\title{
Retraction Note to: Non-peaked Discriminant Analysis for Image Representation
}

Xijian Fan and Qiaolin Ye

\author{
Retraction Note to: \\ Chapter "Non-peaked Discriminant Analysis for Image \\ Representation" in: C. Lee et al. (Eds.): Image and Video \\ Technology, LNCS 11854, \\ https://doi.org/10.1007/978-3-030-34879-3_24
}

The Editors have retracted this chapter [1] because it presents data without authorization for use and contains significant overlap with [2]. Both authors agree to this retraction but not to the wording of the retraction notice.

[1] Fan, X., Ye, Q.: Non-peaked discriminant analysis for image representation. In: Lee, C., Su, Z., Sugimoto, A. (eds.) PSIVT 2019. LNCS, vol. 11854. pp. 310-324. Springer, Cham (2019). https://doi.org/10.1007/978-3-030-34879-3_24

[2] Ye, Q., Li, Z., Fu, L., Zhang, Z., Yang, W., Yang, G.: Nonpeaked discriminant analysis for data representation. IEEE Trans. Neural Networks Learn. Syst. 30(12), 3818-3832 (2019) 